\title{
Acute tuberculous abscess of the thyroid gland
}

\author{
Mohammed Bahgat, ${ }^{1}$ Yassin Bahgat, ${ }^{1}$ Ahmed Bahgat, ${ }^{1}$ Samia Aly ${ }^{2}$ \\ ${ }^{1}$ Department of ENT and Head \& Neck Surgery, Alexandria University Hospitals, Alexandria, Egypt \\ ${ }^{2}$ Department of Pathology, Alexandria University Hospitals, Alexandria, Egypt
}

Correspondence to Dr Mohammed Bahgat, mybahgat18@hotmail.com

\section{Summary}

The authors are presenting our experience of managing an interesting case of a 28-year-old woman who presented to our clinic with a midline neck swelling of 1-month duration. There was a rapid increase in the degree of swelling associated with erythema, pain and fever 7 days before seeking medical help. Plain and contrast-enhanced CT scans of the neck were done. Diagnosis of acute suppurative abscess was made and the patient underwent emergency incision and drainage. Histopathological examination of the abscess wall showed epitheloid and Langhans' giant cells. The findings were suggestive of tuberculosis. Subsequently, a positive culture for Mycobacterium tuberculosis was obtained on Lowenstein-Jenson medium. The patient was started on antituberculous chemotherapy for 6 months. On a 6-month clinical follow-up, the patient was asymptomatic and euthyroid. We discuss the clinical presentation, diagnosis and treatment of this case as well as a review of the literature.

\section{BACKGROUND}

The ability of the thyroid gland to resist infection is well known and infections of this gland are uncommon. ${ }^{1}$ In 1893, Bruns reported the first case of tuberculous thyroiditis. ${ }^{2}$ Since then, there have been relatively few cases of tuberculous involvement of the thyroid gland reported, and almost all cases have been associated with tubercular foci elsewhere in the body. Isolated tuberculosis of the thyroid gland is a rare form of presentation of the disease, and acute tuberculous abscess formation in the thyroid gland is even rarer. ${ }^{3}$ In a study of fine needle aspiration cytology diagnosis of thyroid gland by Das et al, it was found that only $0.6 \%$ of cases were the result of tuberculosis. ${ }^{3}$ Rankin and Graham from the Mayo Clinic reported an incidence rate of tuberculous thyroid in $0.1 \%$ of their patients. $^{4}$

\section{CASE PRESENTATION}

A 28-year-old woman presented with a midline neck swelling of 1-month duration. There was a rapid increase in the degree of swelling associated with erythema, pain and fever 7 days before seeking medical help. There were no symptoms suggestive of hyperthyroidism or hypothyroidism and no breathing or swallowing difficulties were reported. No hoarseness of voice. She had no history of pulmonary or extrapulmonary tuberculosis and she did not have any known contact with tuberculosis. On physical examination there was a tense, erythematous and tender swelling in the lower neck which moved well with deglutition. The margins of the swelling were not well defined. There was marked induration of the skin. There was no palpable lymphadenopathy in the neck. No tracheal shift was detected. Indirect laryngoscopy showed both the vocal cords to be freely mobile.

The erythrocyte sedimentation rate was $50 \mathrm{~mm}$ during the first hour. Thyroid function tests showed a euthyroid state.

\section{INVESTIGATIONS}

Plain and contrast-enhanced CT of the neck showed an irregular, cystic-like mass in the thyroid gland (figure 1). The lesion showed enhancing walls after contrast administration. No tracheal shift was detected. The rest of the gland showed patchy hypodense, non-enhancing areas suggestive of involvement by the disease process. A chest radiograph was normal.

\section{DIFFERENTIAL DIAGNOSIS}

The acute tubercular abscess is difficult to differentiate from an acute suppurative abscess. ${ }^{5} 6$ A definitive diagnosis depends on finding caseating granulomas on the histopathological examination, positive staining for acid-fast bacilli, positive culture of Mycobacterium tuberculosis on Lowenstein-Jensen medium and a prompt therapeutic response to antituberculous drugs. 56

On imaging, the focal form of thyroid tuberculosis is non-specific and is indistinguishable from carcinoma and cystic papillary carcinoma is the main differential diagnosis. Subacute thyroiditis and sarcoidosis are other differential considerations for focal and goitrous types of tuberculosis. ${ }^{7}$

\section{TREATMENT}

After contrast-enhanced CT of the neck, diagnosis of acute suppurative abscess was made and the patient underwent emergency incision and drainage. The abscess cavity continued to discharge pus from the site for some days postprocedure. Histopathological examination of the abscess wall showed epitheloid and Langhans' giant cells. There were areas of caseating necrosis and marked lymphocytic proliferation. A few normal thyroid follicles were seen in the periphery. The findings were suggestive of tuberculosis. Subsequently, a positive culture for $M$ tuberculosis was obtained on Lowenstein-Jenson medium. The patient was started on antituberculous chemotherapy (rifampicin (R), 


\section{BMJ Case Reports}

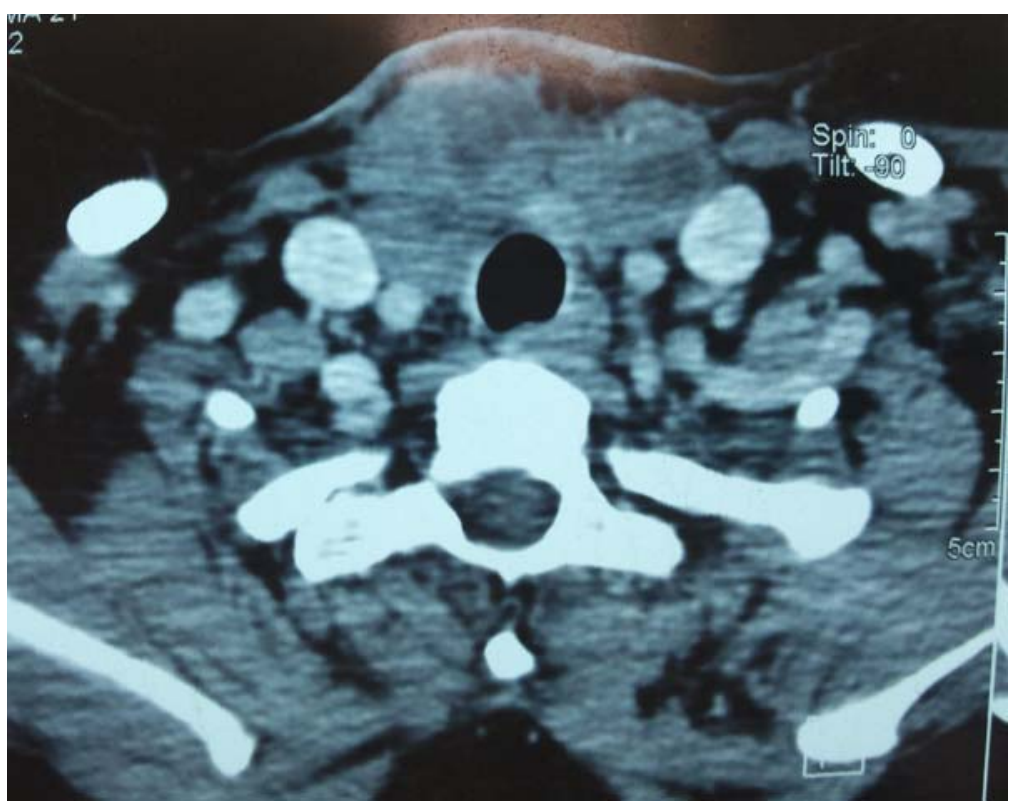

Figure 1 Contrast-enhanced CT scan of the neck (axial view) showing an irregular, cystic-like mass in the thyroid gland.

isoniazid (INH or $\mathrm{H}$ ), ethambutol (E) and pyrazinamide (Z)) for 2 months followed by 4 months of $\mathrm{H}$ and $\mathrm{R}$.

On a 6-month clinical follow-up, the patient was asymptomatic and euthyroid.

\section{OUTCOME AND FOLLOW-UP}

After antituberculous drugs for 6 months, the patient was asymptomatic and euthyroid.

\section{DISCUSSION}

In the 19th century, it was authoritatively suggested that tuberculosis never involved the thyroid gland and that there was some antagonistic action between goitre and tuberculosis. ${ }^{13}$ So that presence of thyroid swelling was a proof that the patient did not have tuberculosis. ${ }^{1}$ The relative immunity of the thyroid gland has been confirmed but has not been explained.

In 1893, Bruns reported the first case of tuberculous thyroiditis. ${ }^{2}$ Since then, there have been relatively few cases of tuberculous involvement of the thyroid gland reported, and almost all cases have been associated with tubercular foci elsewhere in the body. Isolated tuberculosis of the thyroid gland is a rare form of presentation of the disease, and acute tuberculous abscess formation in the thyroid gland is even rarer. ${ }^{3}$ In a study of fine needle aspiration cytology diagnosis of thyroid gland by Das et al, it was found that only $0.6 \%$ of cases were the result of tuberculosis. ${ }^{3}$

Rankin and Graham from the Mayo Clinic reported an incidence rate of tuberculous thyroid in $0.1 \%$ of their patients. ${ }^{4}$ The majority of these cases had a primary focus of tuberculosis elsewhere in the body. ${ }^{4}$ In these cases, the route of infection was usually thought to be haematogenous. ${ }^{4} 5$ In our patient, there was a primary involvement of the thyroid gland by tuberculosis without an overt focus of tuberculosis seen elsewhere in the body.
There are at least five pathological varieties of tuberculosis of the thyroid gland and these are: (1) multiple lesions throughout the gland in association with miliary tuberculosis; (2) a goitre with caseation; (3) cold abscess formation sometimes presenting on the surface of the gland; (4) chronic fibrosing tuberculosis (which is difficult to distinguish from De Quervain's thyroiditis) and (5) acute abscess formation. ${ }^{2}$ Of these, a tuberculous abscess is the least common form of presentation. ${ }^{2-4} 6$

Patients with thyroid tuberculosis might develop recurrent laryngeal nerve palsy as a complication. Some patients can have pressure symptoms on the trachea and oesophagus causing dyspnoea or dysphagia. ${ }^{2}$ Concomitant disorders of the thyroid are not common, but hyperthyroidism and hypothyroidism have been reported. ${ }^{2} 57$ Our patient did not show any evidence of complication as a result of the disease process and was euthyroid.

The difficulty of thyroid tuberculosis is to reach correct diagnosis which is rarely investigated clinically unless there is a chronic discharging sinus from the gland. ${ }^{6}$ Primary focus of tuberculosis, presence of cervical lymphadenopathy, high ESR values and a history of tuberculosis might help with diagnosis, but thyroid tuberculosis can occur in the absence of these features. ${ }^{67}$ A definitive diagnosis depends on finding caseating granulomas on the histopathological examination, positive staining for acid-fast bacilli, positive culture of $M$ tuberculosis on Lowenstein-Jensen medium and a prompt therapeutic response to antituberculous drugs. ${ }^{2}$ 4-6

On imaging, the focal form of thyroid tuberculosis is non-specific and is indistinguishable from carcinoma. Subacute thyroiditis and sarcoidosis are other differential considerations for focal and goitrous types of tuberculosis. ${ }^{7}$ An acute tubercular abscess is difficult to differentiate from an acute suppurative abscess. ${ }^{6} 8$

Treatment of thyroid tuberculosis is mainly medical. Antituberculosis chemotherapy is designed to kill tubercle bacilli rapidly, minimise the potential for the organisms to 
develop drug resistance and sterilise the host's tissues. The achievement of these effects requires combination chemotherapy with specific activities to be administered for a sufficiently long period of time. As a consequence of these effects, the patient is cured and has only a small likelihood of relapse.

The British Thoracic Association demonstrated that a regimen of $\mathrm{H}$ or $\mathrm{INH}$ and $\mathrm{R}$ for 6 months, supplemented during the first 2 months with Z or PZA and E, was as effective as the 9-month regimen of INH and $\mathrm{R}$ with $\mathrm{E}$ in the first 2 months. So the UK and WHO recommendation for tuberculous lymphadenitis is 2 months of INH, R, Z and $\mathrm{E}$ followed by 4 months of INH and R. ${ }^{9} 10$

The US recommendation is 2 months of INH, R, Z and E followed by 7 months of INH and R. ${ }^{9}{ }^{10}$ A Drug Susceptibility Testing should always be performed

\section{Learning points}

- Tuberculosis of the thyroid gland is very rarely reported and almost all cases have been associated with tubercular foci elsewhere in the body. Isolated tuberculosis of the thyroid gland is a rare form of presentation of the disease, and acute tuberculous abscess formation in the thyroid gland is even rarer.

- Patients with thyroid tuberculosis might present with complications like recurrent laryngeal nerve palsy or pressure symptoms on the trachea and oesophagus causing dyspnoea or dysphagia. Concomitant disorders of the thyroid are not common, but hyperthyroidism and hypothyroidism have been reported.

- Diagnosis of thyroid tuberculosis is rarely investigated clinically unless there is a chronic discharging sinus from the gland. Diagnosis can be reached by the presence of primary focus of tuberculosis, presence of cervical lymphadenopathy, high erythrocyte sedimentation rate values and a history of tuberculosis.
- A definitive diagnosis depends on finding caseating granulomas on the histopathological examination, positive staining for acid-fast bacilli, positive culture of Mycobacterium tuberculosis on Lowenstein-Jensen medium and a prompt therapeutic response to antituberculous drugs.

- Treatment of thyroid tuberculosis is mainly medical in the form of comination chemotherapy. The UK and WHO recommendation for tuberculous lymphadenitis is 2 months of isoniazid, rifampicin, ethambutol and pyrazinamide (HREZ) followed by 4 months of HR; the US recommendation is 2 months of HREZ followed by 7 months of isoniazid and rifampicin. A Drug Susceptibility Testing should always be performed.

\section{Competing interests None.}

Patient consent Obtained.

\section{REFERENCES}

1. Parmar H, Hashmi M, Rajput A, et al. Acute tuberculous abscess of the thyroid gland. Australas Radiol 2002;46:186-8.

2. Johnson AG, Phillips ME, Thomas RJS. Acute tuberculous abscess of the thyroid gland. Br J Surg 1993;60:667-9.

3. Kang M, Ojili V, Khandelwal N, et al. Tuberculous abscess of the thyroid gland: a report of two cases. J Clin Ultrasound 2006;34:254-7.

4. Das DK, Pant CS, Chachra KL, et al. Fine needle aspiration cytology diagnosis of tuberculous thyroiditis: a report of eight cases. Acta Cytol 1992; 36:517-22

5. Khalil EA, Elsiddig KE, Elsafi ME, et al. Supra-sternal notch tuberculous abscess: a report of three cases. Trans $R$ Soc Trop Med Hyg 2000;94:58-60.

6. Khan EM, Haque I, Pandey R, et al. Tuberculosis of the thyroid gland: a clinicopathological profile of four cases and review of literature. ANZ J Surg 1993;63:807-10.

7. Terzidis K, Tourli P, Kiapekou E, et al. Thyroid tuberculosis. Hormones (Athens) 2007:6:75-9.

8. Orlandi F, Fiorini S, Gonzatto I, et al. Tubercular involvement of the thyroid gland: a report of two cases. Horm Res 1999;52:291-4.

9. Cook VJ, Manfreda J, Hershfield ES. Tuberculous lymphadenitis in Manitoba: incidence, clinical characteristics and treatment. Can Respir J 2004;11:279-86.

10. Mukherjee S, Sarkar S. Treating tuberculous lymphadenitis—ifs and buts. $\mathrm{J}$ Indian Med Assoc 2003;101:16-17.

This pdf has been created automatically from the final edited text and images.

Copyright 2012 BMJ Publishing Group. All rights reserved. For permission to reuse any of this content visit http://group.bmj.com/group/rights-licensing/permissions.

BMJ Case Report Fellows may re-use this article for personal use and teaching without any further permission

Please cite this article as follows (you will need to access the article online to obtain the date of publication).

Bahgat $\mathrm{M}$, Bahgat $Y$, Bahgat A, Aly S. Acute tuberculous abscess of the thyroid gland. BMJ Case Reports 2012;10.1136/bcr-2012-006906, Published XXX

Become a Fellow of BMJ Case Reports today and you can:

- Submit as many cases as you like

- Enjoy fast sympathetic peer review and rapid publication of accepted articles

- Access all the published articles

- Re-use any of the published material for personal use and teaching without further permission

For information on Institutional Fellowships contact consortiasales@bmjgroup.com

Visit casereports.bmj.com for more articles like this and to become a Fellow 